\title{
Attenuation of Pseudomonas aeruginosa virulence by medicinal plants in a Caenorhabditis elegans model system
}

\section{Correspondence \\ Kalai Mathee \\ Kalai.Mathee@fiu.edu}

Received 7 December 2007

Accepted 19 March 2008

\author{
Allison Adonizio, ${ }^{1}$ Sixto M. Leal, Jr, ${ }^{1}$ Frederick M. Ausubel ${ }^{3}$ \\ and Kalai Mathee ${ }^{1,2}$ \\ ${ }^{1}$ Department of Biological Sciences, College of Arts and Sciences, Florida International University, \\ Miami, FL 33199, USA \\ ${ }^{2}$ Department of Molecular Microbiology and Immunology, College of Medicine, Florida International \\ University, Miami, FL 33199, USA \\ ${ }^{3}$ Department of Genetics, Harvard Medical School, and Department of Molecular Biology, \\ Massachusetts General Hospital, Boston, MA 02114, USA
}

\section{INTRODUCTION}

Pseudomonas aeruginosa is one of the leading pathogens among patients suffering from cystic fibrosis, diffused panbronchitis and chronic obstructive pulmonary disease (Hoiby, 1994; Lieberman, 2003; Registry, 2005). In addition, it remains one of the major causes of nosocomial infections (National Nosocomial Infections Surveillance System, 2004). The success of this organism is attributed to numerous virulence factors (Smith \& Iglewski, 2003b; Tang et al., 1996), its ability to form biofilms (Costerton et al., 1995) and innate antibiotic resistance (De Kievit et al., 2001; Fisher et al., 2005).

Conventional anti-pseudomonal treatment includes elevated doses of $\beta$-lactam, fluoroquinolone or aminoglycoside antibiotics (Hauser \& Sriram, 2005). However, these drugs possess a high degree of toxicity, and mucoid strains of $P$. aeruginosa are rarely eradicated by these treatments (Hauser \& Sriram, 2005; Pedersen, 1992). The failure of existing antibiotics to control infection makes it crucial to find alternatives to currently available drugs. Since pathogenicity in many bacteria is regulated by quorum sensing (QS), or cell-to-cell communication, inhibition of this system can cause attenuation of virulence and protect

Abbreviation: OS, quorum sensing. against infection (Hentzer \& Givskov, 2003; Juhas et al., 2005; Smith \& Iglewski, 2003a).

Plants have evolved numerous chemical strategies for deterring pathogen attack, including the production of bactericidal and anti-infective compounds, leading to their use as medicines (reviewed by Lewis \& Ausubel, 2006). In our previous work, we demonstrated that a number of medicinal plants exhibit anti-QS activity (Adonizio et al., 2006). Extracts of these plants were later shown to have an effect on virulence factor production, biofilm formation, QS gene expression and autoinducer production in $P$. aeruginosa (Adonizio et al., 2008). In this study, we assessed the ability of three plant extracts to attenuate $P$. aeruginosa killing of the nematode Caenorhabditis elegans.

Caenorhabditis elegans is well established as a pertinent and practical model for studying bacterial virulence (Darby et al., 1999; Tan \& Ausubel, 2000), as a number of $P$. aeruginosa factors important in the killing of Caenorhabditis elegans are also relevant to mammalian systems (Rahme et al., 1995; Tan et al., 1999a). 'Fast killing' of Caenorhabditis elegans by P. aeruginosa strain PA14 (on rich media) is mediated through the production of virulence factors such as phenazines, whereas 'slow killing' (on minimal media) occurs via ingestion of the bacteria and subsequent infection (Mahajan-Miklos et al., 1999; 
Tan et al., 1999a, b). The related strain PAO1 causes death through cyanide poisoning and neuromuscular paralysis (Gallagher \& Manoil, 2001). Importantly, P. aeruginosamediated killing of Caenorhabditis elegans in all three of these cases is dependent in part on the QS system. Thus addition of plant compounds that have an effect on $P$. aeruginosa $\mathrm{QS}$ should attenuate virulence factor production and the subsequent death of Caenorhabditis elegans.

The advantage of using a live animal model when screening for anti-infective compounds is that both the efficacy and the host toxicity of a plant extract can be tested concurrently. In this report, we show that extracts from three different plant species caused a marked decrease in $P$. aeruginosa-mediated killing of Caenorhabditis elegans without affecting worm fitness on Escherichia coli. This approach can be expanded to the screening of natural product libraries or native extract sources.

\section{METHODS}

Preparation of plant extracts. Samples of Conocarpus erectus (Combretaceae), Callistemon viminalis (Myrtaceae) and Bucida buceras (Combretaceae) were collected and processed according to methods described previously (Adonizio et al., 2006). Briefly, pulverized plant material was extracted in boiling water, freeze-dried using a lyophilizer and stored at $-20{ }^{\circ} \mathrm{C}$ until needed. Lyophilized extracts were reconstituted in a small volume of sterile water and added to molten agar at a final concentration of $1 \mathrm{mg} \mathrm{ml}^{-1}$. This concentration was chosen based on original dose-dependence studies and subsequent analysis of the extracts against $P$. aeruginosa (Adonizio et al., 2006, 2008). This also allowed comparison of experiments in this paper with those in previous studies.

Bacterial strains and growth conditions. Because the tested extracts have been shown to affect the bacterial QS system (Adonizio et al., 2006, 2008), the QS mutant $\Delta l a s R$ was used as a reference strain. Wild-type P. aeruginosa PAO1 (Holloway \& Morgan, 1986) and its isogenic mutant PAO1 LlasR (Gambello \& Iglewski, 1991) were used in the paralytic assay. Wild-type P. aeruginosa PA14 (Rahme et al., 1995) and its PA14 LlasR (Liberati et al., 2006) were used in the slowkilling and fast-killing assays. E. coli OP50 was used as the control in all assays, as this strain is the standard laboratory food of Caenorhabditis elegans (Brenner, 1974). All bacterial strains were grown overnight in Luria-Bertani broth at $37{ }^{\circ} \mathrm{C}$ and transferred to plates, depending on the required conditions.

Nematode culture. The wt Caenorhabditis elegans (Bristol) N2 hermaphrodite strain was used in this study (Brenner, 1974). Worms were synchronized by hypochlorite treatment of gravid adults, hatching of the eggs overnight in M9 minimum buffer (Brenner, 1974) and plating L1-stage worms onto lawns of $E$. coli on nematode growth medium plates (Brenner, 1974). Synchronized worms were grown to the L4 or young adult stage at $25{ }^{\circ} \mathrm{C}$ for use in the killing assays.

Caenorhabditis elegans paralytic assay. Brain heart infusion agar plates with or without plant extract were seeded with $10 \mu \mathrm{l}$ of an overnight culture of $E$. coli OP50 or $P$. aeruginosa PAO1 or PAO1 1 lasR and incubated at $37{ }^{\circ} \mathrm{C}$ for $24 \mathrm{~h}$ to form lawns of bacteria (Darby et al., 1999). Nematodes were washed off stock plates and suspended in a minimal volume of M9 buffer ( $\mathrm{pH}$ 6.5). Droplets containing 20-40 adult nematodes were placed onto the bacterial lawns and the plates were incubated at room temperature $\left(21-23{ }^{\circ} \mathrm{C}\right)$.
Worms were evaluated for viability every hour for a total of $4 \mathrm{~h}$. Worms were scored as dead when they no longer responded to physical stimuli.

Caenorhabditis elegans fast-killing assay. Fast-killing plates (peptone/glucose medium with $0.15 \mathrm{M}$ sorbitol; Mahajan-Miklos et al., 1999) with or without plant extract were seeded with $10 \mu \mathrm{l}$ of an overnight culture of OP50, PA14 or PA14 LlasR. Plates were incubated for $24 \mathrm{~h}$ at $37^{\circ} \mathrm{C}$ and then at room temperature $\left(21-23{ }^{\circ} \mathrm{C}\right)$ for another $12 \mathrm{~h}$. Approximately 20 L4-stage Caenorhabditis elegans were transferred with a wire pick onto plates at this time. Worms were evaluated for viability every hour for a total of $4 \mathrm{~h}$. As in the previous assay, worms were considered dead when they no longer responded to physical stimuli.

Caenorhabditis elegans slow-killing assay. Slow-killing plates (modified nematode growth medium; Tan et al., 1999b) with or without plant extract were seeded with $10 \mu \mathrm{l}$ of an overnight culture of OP50, PA14 or PA14 LlasR. Plates were incubated for $24 \mathrm{~h}$ at $37{ }^{\circ} \mathrm{C}$ and then at room temperature $\left(21-23{ }^{\circ} \mathrm{C}\right)$ for another $24 \mathrm{~h}$. Approximately 20 L4-stage Caenorhabditis elegans were transferred onto plates at this time. Worms were evaluated for viability every 2 $4 \mathrm{~h}$ for a total of $58 \mathrm{~h}$. As in the previous assays, worms were considered dead when they no longer responded to physical stimuli.

Statistics and reproducibility. All experiments were performed in triplicate. Killing curves represent the mean of three separate experiments. Data were analysed using one-way analysis of variance with a $P$ value of 0.05 using the statistical software package sPss. As the starting number of worms was different in each case, the percentage of worms still alive rather than the actual number of worms was used for comparison.

\section{RESULTS AND DISCUSSION}

In this study, we assess the potential of extracts from Conocarpus erectus, Callistemon viminalis and B. buceras to reduce nematode death resulting from $P$. aeruginosa infection. Prior work on these plants revealed an effect on the bacterial QS system (Adonizio et al., 2006, 2008); thus the QS mutant $\Delta l a s R$ was used as a reference strain. Although there is some precedence for testing plant extracts in a nematode model system (Rasmussen et al., 2005), this is the first study to evaluate a statistically significant number of worms in both toxin and infectionbased assays.

\section{Medicinal plants rescue paralytic killing of Caenorhabditis elegans by $\boldsymbol{P}$. aeruginosa}

Previous studies have shown that QS signalling is required for maximum levels of worm killing (Mahajan-Miklos et al., 1999; Tan et al., 1999b). Thus the success of these plant extracts against $P$. aeruginosa $\mathrm{PAO} 1$ in terms of blocking QS signalling (Adonizio et al., 2008) suggested that they might also reduce death in a PAO1-nematode model. Approximately $50 \%$ of the worms died between 1 and $2 \mathrm{~h}$ after transfer to PAO1, with all nematodes dead after $4 \mathrm{~h}$ (Fig. 1a). In contrast, all of the worms on E. coli OP50 remained alive throughout the assay (not shown). As expected, the QS mutant PAO1 1 lasR showed reduced nematode death, with $85 \%$ alive between 1 and $2 \mathrm{~h}$ and 


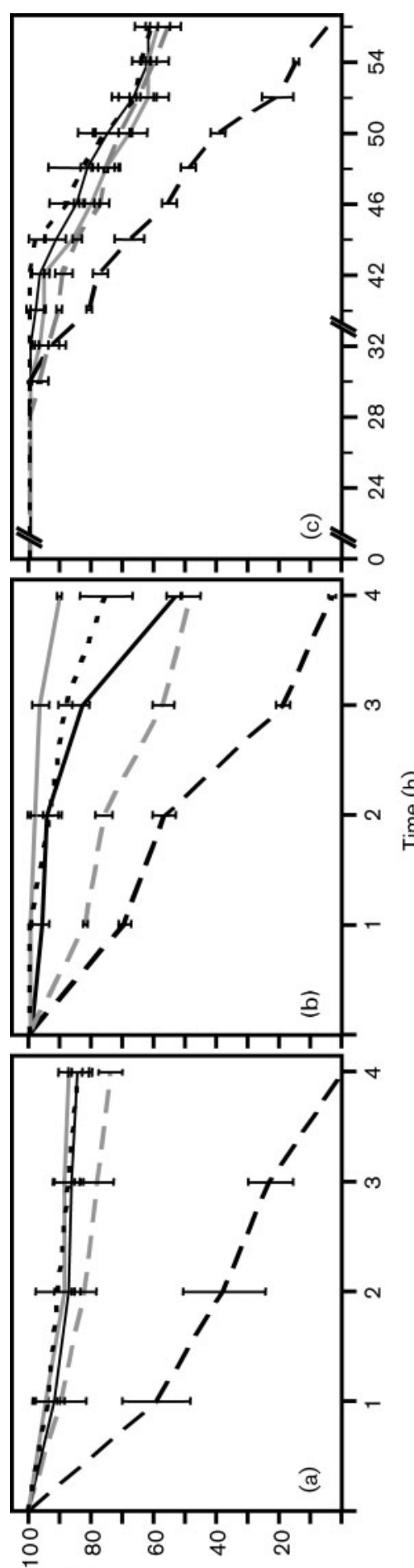

(\%) әм!|е sәроцешә N

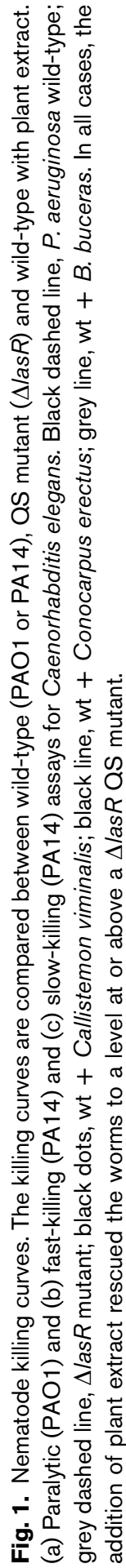

$74 \%$ still alive at $4 \mathrm{~h}$ (Fig. 1a). After $4 \mathrm{~h}$, approximately 85 , 84 and $87 \%$ of the worms were alive on PAO1 plates that contained Conocarpus erectus, Callistemon viminalis and $B$. buceras extracts, respectively (Fig. 1a). In this and all other assays in this study, there was no observable effect of the added plant extracts on worm survival, lifespan or brood size on E. coli OP50 (not shown), indicating a lack of toxicity of the compounds.

All three of the plant extracts, when added to plates containing PAO1, suppressed killing to a level greater than the QS mutant (Fig. 1a), i.e. there was a significant difference compared with PAO1DlasR and the extractcontaining plates $(P<0.05$ in all cases $)$, but not among individual extracts at the end of the assay. All extract plates and the $\Delta$ lasR mutant were significantly different from wild-type PAO1 without treatment.

Death via PAO1 is due to cyanide asphyxiation and paralysis of Caenorhabditis elegans (Gallagher \& Manoil, 2001). The $h c n$ operon in $P$. aeruginosa mediates cyanide production and is controlled by the QS regulators LasR and RhlR (Pessi $\&$ Haas, 2000). Attenuation of virulence and nematode mortality has been shown with both $\Delta$ lasR (Darby et al., 1999) and $\Delta h c n$ (Gallagher \& Manoil, 2001) strains. Thus the results from the paralytic assay suggested that the addition of these extracts was affecting the production of cyanide either through $h c n$ directly or indirectly via the QS genes. The latter hypothesis agrees with our previous in vitro analysis in PAO1 (Adonizio et al., 2008), which showed a significant reduction in lasR and rhlR gene activity by these extracts.

\section{Medicinal plants prevent fast killing of Caenorhabditis elegans by $\boldsymbol{P}$. aeruginosa}

The results of the fast-killing assay are shown in Fig. 1(b). On PA14, approximately $50 \%$ of the worms were dead within $2 \mathrm{~h}$, with all worms dead by $4 \mathrm{~h}$. At this point, all of the worms on OP50 were still alive (not shown). Again, the QS mutant (PA14 $\Delta$ lasR) reduced nematode death, with $75 \%$ alive between 1 and $2 \mathrm{~h}$ and $47 \%$ alive at $4 \mathrm{~h}$. At the end of the assay, approximately 53, 75 or $90 \%$ of the worms were alive on PAO1 plates with added Conocarpus erectus, Callistemon viminalis and B. buceras extract, respectively (Fig. 1b). As with PAO1, all three of the plant extracts suppressed killing at or above the level of the QS mutant. There was no significant difference between PA14 $\operatorname{las} R$ and the Conocarpus erectus extract plates at $4 \mathrm{~h}(P>0.05)$; however, the Callistemon viminalis and B. buceras extracts were significantly better at preventing worm death $(P<0.01$ in both cases). Extract-containing plates were all significantly different from each other, but still showed a large reduction in nematode death when compared with wild-type PA14 without treatment. Overall, there was a pronounced inhibitory effect of the plant extracts on P. aeruginosa PA14 fast killing of Caenorhabditis elegans.

Fast killing of Caenorhabditis elegans is mediated by the production of virulence factors such as phenazines 
(Mahajan-Miklos et al., 1999). A $\Delta p h n A p h n B$ deletion mutant has been shown to abolish nematode death completely, whilst a TnphoA mutation of the related gene $p h z B$ has been shown to greatly reduce mortality in mice and Arabidopsis (Mahajan-Miklos et al., 1999). Like many virulence factors, phenazines are partially under the control of the QS gene rhlR (Brint \& Ohman, 1995; Latifi et al., 1995). The results from the PA14 fast-killing assay suggested that the addition of extracts affected phenazine production, either directly through the $p h z$ and $p h n$ genes or indirectly through the QS system via rhlR. All three extracts were shown previously to significantly affect the $r h l I / R$ system; however, Conocarpus erectus has less of an effect on $\mathrm{N}$-acylhomoserine lactone production and biofilm formation (strain PAO1; Adonizio et al., 2008) than either B. buceras or Callistemon viminalis. Although still successful, Conocarpus erectus was less efficient in preventing nematode death in the fast-killing assay than the other extracts.

\section{Plant extracts reduce the mortality of Caenorhabditis elegans due to slow killing by $\boldsymbol{P}$. aeruginosa}

The slow-killing assay left $50 \%$ of nematodes dead on PA14 between 48 and $50 \mathrm{~h}$, with all worms dead by $58 \mathrm{~h}$ (Fig. 1c). The control worms on E. coli OP50 remained alive throughout the assay (not shown). The QS mutant (PA14_lasR) reduced nematode death, with $75 \%$ alive between 48 and $50 \mathrm{~h}$ and $53 \%$ alive at $58 \mathrm{~h}$. At this time, approximately 60,59 and $57 \%$ of worms were alive on PA14 plates with added Conocarpus erectus, Callistemon viminalis and B. buceras extract, respectively (Fig. 1c). All three of the plant extracts, when added to plates containing wild-type PA14, suppressed killing to the level of the QS mutant. There was no significant difference between PA14 1 lasR and the extract plates or between individual extracts at $58 \mathrm{~h}(P>0.05$ in all cases $)$; however, all extracts were significantly different from PA14 without treatment, suggesting a marked effect of the plant extracts on $P$. aeruginosa infection of Caenorhabditis elegans.

Slow killing of Caenorhabditis elegans occurs over approximately $60 \mathrm{~h}$ due to ingestion of and subsequent infection by $P$. aeruginosa (Tan et al., 1999a). Nematode mortality is attenuated by TnphoA mutations of lasR and gacA (Tan et al., 1999b), suggesting that QS is required for the infection process. The addition of plant extracts in this assay drastically reduced nematode death, suggesting an effect on lasR or gacA. Previous work on these extracts corroborated the inhibitory effect on lasR; however, the effect on gacA was not tested directly (Adonizio et al., 2008). An effect on either of these factors remains a plausible hypothesis.

\section{Conclusions}

The three plant extracts from Conocarpus erectus (Combretaceae), Callistemon viminalis (Myrtaceae) and $B$. buceras (Combretaceae), in all three assays, showed a highly significant reduction in virulence when compared with wildtype PAO1 and PA14 without treatment. Overall, the tested plant extracts reduced nematode death by approximately 60 $90 \%$ on wild-type $P$. aeruginosa. In each case, this reduction was equal to or greater than that of the corresponding QS mutant strain. The fact that the plant extracts reduced virulence across the board suggests that they are possibly affecting an upstream QS gene such as las or rhl, or perhaps a global regulator such as GacA. This further corroborates our previous data on the anti-QS effect of these plant extracts (Adonizio et al., 2008). All extracts inhibited nematode death without any significant bactericidal effect, leaving QS inhibition as a plausible hypothesis. In addition, none of the tested plants showed any toxicity in the nematode model, making them reasonable candidates for purification and drug development.

Conocarpus erectus, B. buceras and Callistemon viminalis (and other closely related species) have been used medicinally to treat bacterial infections either as teas or as poultices (Burkhill, 1985; Irvine, 1961; Melendez, 1982; Morton, 1981; Stewart \& Percival, 1997). Thus the plants were extracted with hot water to provide greater congruity with traditional preparation methods. Although teas and poultices are many steps removed from modern formulae, traditional use suggests the potential success of topical or enteral routes of administration.

With the increase in bacterial resistance to antibiotics, we should look to the past in the hope of finding solutions for the future. Plants have been used medicinally for thousands of years and, even without marked antibiotic activity, these three plants are still efficacious in ameliorating disease. We have previously shown the activity of these plants on $P$. aeruginosa alone and, although the exact mechanism of action is not yet known, the nematode experiments described in this paper are consistent with their previous and potential further use as anti-infectives.

\section{ACKNOWLEDGEMENTS}

The authors gratefully acknowledge the support of the National Institute of Health, National Center for Alternative and Complementary Medicine (NCCAM) NRSA \#1-T32-AT01060-01 (A.A. and K.M.), NCCAM 1-R15-AT002626-01 (K.M.), National Institute of General Medical Sciences (NIGMS) Research Initiative for Scientific Enhancement (RISE) program grant R25 GM61347 (A. A.), National Institute of Allergy and Infectious Diseases (NIAID) R01 AI072508 (F.M.A.), NIAID R01 AI064332 (F. M.A.) and the Cystic Fibrosis Foundation \#ADONIZO06H0 (A.A.). We also thank the members of the Mathee and Ausubel laboratories for assistance and support, especially Terry Moy and Rhonda Feinbaum.

\section{REFERENCES}

Adonizio, A. L., Downum, K., Bennett, B. C. \& Mathee, K. (2006). Anti-quorum sensing activity of medicinal plants in southern Florida. J Ethnopharmacol 105, 427-435. 
Adonizio, A., Kong, K.-F. \& Mathee, K. (2008). Inhibition of quorum sensing-controlled virulence factor production in Pseudomonas aeruginosa by South Florida plant extracts. Antimicrob Agents Chemother 52, 198-203.

Brenner, S. (1974). The genetics of Caenorhabditis elegans. Genetics 77, 71-94.

Brint, J. M. \& Ohman, D. E. (1995). Synthesis of multiple exoproducts in Pseudomonas aeruginosa is under the control of RhlR-RhlI, another set of regulators in strain PAO1 with homology to the autoinducerresponsive LuxR-LuxI family. J Bacteriol 177, 7155-7163.

Burkhill, H. M. (1985). The Useful Plants of West Tropical Africa. London: Kew Royal Botanic Gardens.

Costerton, J. W., Lewandowski, Z., Caldwell, D. E., Korber, D. R. \& Lappin-Scott, H. M. (1995). Microbial biofilms. Annu Rev Microbiol 49, 711-745.

Darby, C., Cosma, C. L., Thomas, J. H. \& Manoil, C. (1999). Lethal paralysis of Caenorhabditis elegans by Pseudomonas aeruginosa. Proc Natl Acad Sci U S A 96, 15202-15207.

De Kievit, T. R., Parkins, M. D., Gillis, R. J., Srikumar, R., Ceri, H., Poole, K., Iglewski, B. H. \& Storey, D. G. (2001). Multidrug efflux pumps: expression patterns and contribution to antibiotic resistance in Pseudomonas aeruginosa biofilms. Antimicrob Agents Chemother 45, 1761-1770.

Fisher, J. F., Meroueh, S. O. \& Mobashery, S. (2005). Bacterial resistance to $\beta$-lactam antibiotics: compelling opportunism, compelling opportunity. Chem Rev 105, 395-424.

Gallagher, L. A. \& Manoil, C. (2001). Pseudomonas aeruginosa PAO1 kills Caenorhabditis elegans by cyanide poisoning. J Bacteriol 183, 6207-6214.

Gambello, M. J. \& Iglewski, B. H. (1991). Cloning and characterization of the Pseudomonas aeruginosa lasR gene, a transcriptional activator of elastase expression. J Bacteriol 173, 3000-3009.

Hauser, A. R. \& Sriram, P. (2005). Severe Pseudomonas aeruginosa infections. Tackling the conundrum of drug resistance. Postgrad Med 117, 41-48.

Hentzer, M. \& Givskov, M. (2003). Pharmacological inhibition of quorum sensing for the treatment of chronic bacterial infections. J Clin Invest 112, 1300-1307.

Hoiby, N. (1994). Diffuse panbronchiolitis and cystic fibrosis: East meets West. Thorax 49, 531-532.

Holloway, B. W. \& Morgan, A. F. (1986). Genome organization in Pseudomonas. Annu Rev Microbiol 40, 79-105.

Irvine, F. R. (1961). Woody Plants of Ghana. London: Oxford University Press.

Juhas, M., Eberl, L. \& Tummler, B. (2005). Quorum sensing: the power of cooperation in the world of Pseudomonas. Environ Microbiol 7, 459-471.

Latifi, A., Winson, M. K., Foglino, M., Bycroft, B. W., Stewart, G. S., Lazdunski, A. \& Williams, P. (1995). Multiple homologues of LuxR and LuxI control expression of virulence determinants and secondary metabolites through quorum sensing in Pseudomonas aeruginosa PAO1. Mol Microbiol 17, 333-343.

Lewis, K. \& Ausubel, F. M. (2006). Prospects for plant-derived antibacterials. Nat Biotechnol 24, 1504-1507.

Liberati, N. T., Urbach, J. M., Miyata, S., Lee, D. G., Drenkard, E., Wu, G., Villanueva, J., Wei, T. \& Ausubel, F. M. (2006). An ordered, nonredundant library of Pseudomonas aeruginosa strain PA14 transposon insertion mutants. Proc Natl Acad Sci U S A 103, 2833-2838.

Lieberman, D. (2003). Pseudomonal infections in patients with COPD: epidemiology and management. Am J Respir Med 2, 459-468.

Mahajan-Miklos, S., Tan, M. W., Rahme, L. G. \& Ausubel, F. M. (1999). Molecular mechanisms of bacterial virulence elucidated using a Pseudomonas aeruginosa-Caenorhabditis elegans pathogenesis model. Cell 96, 47-56.

Melendez, E. N. (1982). Plantas Medicinales de Puerto Rico: Folklore y Fundamentos Científicos. Río Piedras, PR: Editorial de la Unversidad de Puerto Rico.

Morton, J. F. (1981). Atlas of Medicinal Plants of Middle America: Bahamas to Yucatan. Springfield, IL: Charles C. Thomas.

National Nosocomial Infections Surveillance System (2004). National Nosocomial Infections Surveillance (NNIS) system report, data summary from January 1992 through June 2004: a report from the NNIS System. Am J Infect Control 32, 470-485.

Pedersen, S. S. (1992). Lung infection with alginate-producing, mucoid Pseudomonas aeruginosa in cystic fibrosis. APMIS Suppl 28, $1-79$.

Pessi, G. \& Haas, D. (2000). Transcriptional control of the hydrogen cyanide biosynthetic genes $h c n \mathrm{ABC}$ by the anaerobic regulator ANR and the quorum-sensing regulators LasR and RhlR in Pseudomonas aeruginosa. J Bacteriol 182, 6940-6949.

Rahme, L. G., Stevens, E. J., Wolfort, S. F., Shao, J., Tompkins, R. G. \& Ausubel, F. M. (1995). Common virulence factors for bacterial pathogenicity in plants and animals. Science 268, 1899-1902.

Rasmussen, T. B., Bjarnsholt, T., Skindersoe, M. E., Hentzer, M., Kristoffersen, P., Kote, M., Nielsen, J., Eberl, L. \& Givskov, M. (2005). Screening for quorum-sensing inhibitors (QSI) by use of a novel genetic system, the QSI Selector. J Bacteriol 187, 1799-1814.

Registry (2005). Patient Registry 2004 Annual Report. Bethesda, MD: Cystic Fibrosis Foundation.

Smith, R. S. \& Iglewski, B. H. (2003a). Pseudomonas aeruginosa quorum sensing as a potential antimicrobial target. J Clin Invest 112, 1460-1465.

Smith, R. S. \& Iglewski, B. H. (2003b). Pseudomonas aeruginosa quorum-sensing systems and virulence. Curr Opin Microbiol 6, 56-60.

Stewart, K. \& Percival, B. (1997). Bush Foods of New South Wales: a Botanic Record and an Aboriginal Oral History. Sydney: Royal Botanic Gardens.

Tan, M. W. \& Ausubel, F. M. (2000). Caenorhabditis elegans: a model genetic host to study Pseudomonas aeruginosa pathogenesis. Curr Opin Microbiol 3, 29-34.

Tan, M. W., Mahajan-Miklos, S. \& Ausubel, F. M. (1999a). Killing of Caenorhabditis elegans by Pseudomonas aeruginosa used to model mammalian bacterial pathogenesis. Proc Natl Acad Sci U S A 96, 715-720.

Tan, M. W., Rahme, L. G., Sternberg, J. A., Tompkins, R. G. \& Ausubel, F. M. (1999b). Pseudomonas aeruginosa killing of Caenorhabditis elegans used to identify $P$. aeruginosa virulence factors. Proc Natl Acad Sci U S A 96, 2408-2413.

Tang, H. B., DiMango, E., Bryan, R., Gambello, M., Iglewski, B. H., Goldberg, J. B. \& Prince, A. (1996). Contribution of specific Pseudomonas aeruginosa virulence factors to pathogenesis of pneumonia in a neonatal mouse model of infection. Infect Immun 64, 37-43. 